\title{
Development of thymic lymphomas in mice disrupted of Brca2 allele in the thymus
}

\author{
Pil-Gu Park ${ }^{1}$ and Hyunsook Lee ${ }^{1,2}$ \\ ${ }^{1}$ Research Center for Functional Cellulomics \\ Department of Biological Sciences and \\ Institute of Molecular Biology and Genetics \\ Seoul National University \\ Seoul 151-742, Korea \\ ${ }^{2}$ Corresponding author: Tel, 82-2-880-9121; \\ Fax, 82-2-886-4335; E-mail, HL212@ snu.ac.kr
}

\section{Accepted 19 March 2008}

Abbreviations: DSB, double-strand DNA break; MEF, mouse embryonic fibroblast; LOH, loss-of-heterozygosity

\begin{abstract}
Germ-line mutations in BRCA2 predispose to early-onset cancer. Homozygous mutant mouse, which has Brca2 truncated in exon 11 exhibit paradoxic occurrence of growth retardation and development of thymic lymphomas. However, due to its large embryonic lethality, cohort studies on the thymic lymphomas were not feasible. With the aid of Cre-loxP system, we demonstrate here that thymus-specific disruption of Brca2 allele without crossing it to p53-mutant background leads to the development of thymic lymphomas. Varying from 16 weeks to 66 weeks after birth, $25 \%$ of mice disrupted of Brca2 in the thymus died of thymic lymphomas, whereas previous report did not observe lymphomagenesis using similar Cre-loxP system. Future analysis of thymic lymphomas from these mice presented here will provide information on the cooperative mutations that are required for the BRCA2-associated pathogenesis of cancer.
\end{abstract}

Keywords: BRCA2; mice; knockout; Cre recombinase; lymphoma; $T$ cells

\section{Introduction}

Individuals who inherit one mutant allele of $B R C A 2$ are predisposed to early-onset breast cancer (Wooster et al., 1994; Gayther et al., 1997). Studies on the functions of BRCA2 revealed that it was invoIved in double-strand DNA break (DSB) repair via binding to Rad51 recombinase (Patel et al., 1998;
Venkitaraman, 2003, 2004). Involvement of BRCA2 in DSB repair has been first evidently revealed by metaphase chromosome spreads: Brca2-deficient mouse embryonic fibroblasts (MEFs) manifest chromosome structure instability such as aberrant chromosome breaks, exchanges, and random translocations because they lack the ability to repair spontaneous DNA lesions (Patel et al., 1998). Following studies succeeded in demonstrating the mechanism by which BRCA2 regulates Rad51 and DNA repair using in vitro biochemical studies (Davies and Pellegrini, 2007) and structural studies as well (Pellegrini et al., 2002; Yang et al., 2002; Huang et al., 2006).

Notably, chromosome instability manifested by the dysfunctional BRCA2 is not restricted to chromosome structure instability but involves chromosome number instability, aneuploidy, as well, suggesting that it possess multiple functions including cell cycle regulation in order to keep the genome intact. Indeed studies in vivo suggest that BRCA2 participates in embryonic development (Suzuki et al., 1997), regulation of T cell apoptosis (Cheung et al., 2002), mitotic cell cycle regulation (Lee et al., 1999; Lee, 2003), and cytokinesis (Daniels et al., 2004). Given the multiple roles in vivo, understanding the pathogenesis of BRCA2-mutated tumorigenesis inevitably requires detailed studies at the animal level.

In order to understand BRCA2-associated cancer development in vivo, several kinds of mouse models were designed. First, targeted disruption of BRCA2 allele resulted in embryonic lethality at embryonic day 6.5 (Ludwig et al., 1997; Sharan et al., 1997; Suzuki et al., 1997). The large exon11 is conserved between mouse and human. Importantly, this region is frequently mutated in ovarian cancers. When exon11 is targeted, embryonic survival was prolonged and some mice survived to birth. These mice exhibited paradoxic occurrence of growth retardation and thymic lymphoma; they were smaller in size compared to wild-type and succumbed to death at 12 weeks after weaning due to development of thymic lymphoma (Connor et al., 1997; Friedman et al., 1998). Notably, exon 11 contains 8 $B R C$ repeats that are capable of binding to Rad51 and maintains monomeric Rad51 (Davies et al., 2001; Pellegrini et al., 2002). Mice which survived to birth retained at least three BRC repeats, which are considered to have a partial activity in Rad51-binding, indicating that the mutation in these 
mice is hypomorphic. Although the embryonic survival was prolonged and some mice survived to birth, the numbers of surviving Brca $2^{\operatorname{Tr}}$ mice were small and therefore analyses on the lymphomas were limited.

In order to overcome these limitations, Bern's group succeeded in generating conditional knockout mice using Cre-loxP system (Jonkers et al., 2001). In this mouse, exon 11 of Brca2 is flanked by loxP sites, which was also the targeting site for Brca2 ${ }^{\mathrm{Tr}}$ mice that developed tumor (Friedman et al., 1998). With this conditional knockout mouse model, they recapitulated and confirmed the previous study (Lee et al., 1999) that p53 inactivation is required for BRCA2-mutant cells to develop epithelial cancer. Using similar loxP-floxed Brca2 allele, another group studied on the role of BRCA2 in the thymus. The result showed that $B R C A 2$ regulated $T$ cell apoptosis but they failed to detect $T$ cell lymphoma unless they were crossed to p53-mutant background (Cheung et al., 2002).

Despite the low cohort, all the Brca2 ${ }^{T r}$ mice that survived to birth developed thymic lymphoma (Friedman et al., 1998). Because bigger cohort study on Brca2-disrupted $\mathrm{T}$ cell lymphomas and analysis on $\mathrm{T}$ cell lymphomas at the molecular level was required, we generated mice that were conditionally disrupted of Brca2 allele in the thymus. For this aim, we crossed Brca2 ${ }^{\mathrm{F} 11}$ (Jonkers et al., 2001) mice with Lck-Cre mice. Mouse positive for both $B r c a 2^{F 11}$ allele and Cre $\left(B R C A 2^{F 11 / F 11 ;}\right.$; Lck-Cre) will lose Brca2 in the thymus. Here, we report the tumor formation, their incidence and analysis on the cultured thymic lymphoma. The $\mathrm{T}$ cell lymphomas derived from Brca2- deficient thymus will be of valuable source in understanding the molecular mechanism of tumorigenesis in Brca2-deficient background.

\section{Materials and Methods}

\section{Animal experiments}

To generate mice that were disrupted of Brca2 allele specifically in the thymus, Brca $2^{\mathrm{F}^{11}}$ mice (Jonkers et al., 2001) were crossed with Lck-Cre mice. Through continuous mating and screening using PCR, statistically meaningful number of each line were collected and observed for their phenotype. Each mice were observed everyday, and when they died, they were examined immediately and performed series of experiments. All experiments performed were approved by Institutional Animal Care and Use Committees (IACUC) of Seoul National University. We strictly followed the guidelines of Policy and Regulation for the Care and Use of Laboratory Animals.

\section{PCR genotyping}

Mouse tail for genotyping was cut and incubated with tail-lysis Buffer [50mM Tris, 100 mM EDTA, 1\% SDS, $200 \mu \mathrm{g} / \mathrm{ml}$ of Proteinase K (Sigma)] for $16 \mathrm{~h}$ in $55^{\circ} \mathrm{C}$. Supernatants were separated by centrifugation and phenol-chloroform purified. Four different primers were used to screen loxP-flanked alleles. Primer 10F (GGCTGTCTTAGAACTTAGGCTG) and primer 10R (CTCCACACATACATCATGTGTC) are at the 10th intron of BRCA2. Primer 11F (CTCATCATTTGTTGCCTCACTTC) and 11R (TGTTGGATACAAGGCATGTACAC) locate at intron 11 of BRCA2. For screening Lck-Cre mice, Cre-5 primer (CGATGCAACGAGTGATGAGGTTC) and Cre-3 primer (GCACGTTCACCGGCATCAAC) were used (Jonkers et al., 2001).

\section{Isolation and culture of $T$ cells from thymus}

In order to analyze $T$ cells from thymus, thymuses of control mice and Brca2 ${ }^{\mathrm{F} 11 / \mathrm{F} 11}$ mice were surgically isolated. Thymus was flushed several times with RPMI supplemented with $10 \%$ FBS and $50 \mu \mathrm{M}$ of $\beta$-mercaptoethanol using $2 \mathrm{ml}$ syringe without needle. Then the cells flushed from the thymus were cultured in the media (RPMI, supplemented with $10 \%$ FBS, $50 \mu \mathrm{M}$ of $\beta$-mercaptoethanol) provided with recombinant IL-2 (BioLegend, San Diego) and IL-7 (BioLegend, San Diego) before analysis for flow cytometry.

\section{Flow cytometric analysis}

To analyze CD4 and CD8 populations, T cells adducts from dead mice were mashed. $10^{6}$ cells of mashed cellular adducts were stained with FITCconjugated mouse monoclonal CD4 antibody (BioLegend, San Diego) and PE-conjugated mouse monoclonal CD8b antibody (BioLegend, San Diego) for $45 \mathrm{~min}$ at $4^{\circ} \mathrm{C}$. Stained cells were processed by FACSCalibur (BD Biosciences, NJ) and analyzed with CellQuest Program (BD Biosciences, NJ).

\section{Results}

\section{T cell-specific disruption of Brca2 allele}

$B R C A 2^{F 11}$ allele has two loxP sites flanking exon 11. Only when Cre recombinase is expressed, it would induce the excision of exon 11 in this allele. Otherwise, the inserted loxP sites would not harm the allele (Figure 1A). Primers were designed to distinguish the excised exon 11 from wild-type allele 
or the unexcised loxP-flanked allele (Jonkers et al., 2001). Using the PCR screening method with the primers indicated, we were able to genotype for Brca2 allele. Brca2 $2^{F 11 /+}$ mice were crossed with Lck-Cre transgenic mice to specifically excise Brca2 allele in thymic $T$ cells. PCR screening showed that Brca2 ${ }^{F 11}$ allele could be disrupted and analyzed (Figure 1B). Since we were specifically interested in tumor formation by disrupting Brca2, we chose to backcross the mice for over 10 generations to FvB strain, which is comparatively tumor-prone (Woodworth et al., 2004). Lck-Cre mice, which express Cre recombinase that recognizes loxP sites only in thymus, were also backcrossed to FvB for more than 10 generations. Through continuous mating and screening by PCR (Figure 1B), we collected statistically meaningful number of each mouse line and observed for their phenotype.

\section{Reduced survival rate in $B R C A 2^{F 11 / F 11}$; Lck-Cre mice compared to control mice}

In humans, tumors develop with the loss-of-heterozygosity (LOH). In mouse models for cancer, $\mathrm{LOH}$ is rarely observed. Because $\mathrm{LOH}$ of tumor su-

\section{A} WT BRCA2 allele

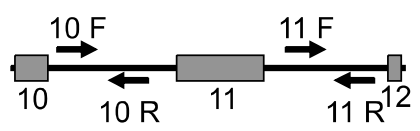
$B R C A 2^{F 11}$ allele

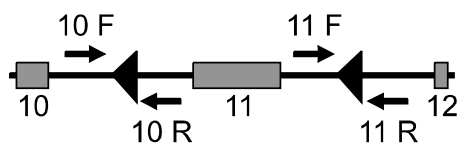

$$
B R C A 2^{D e / 11} \text { allele }
$$

B

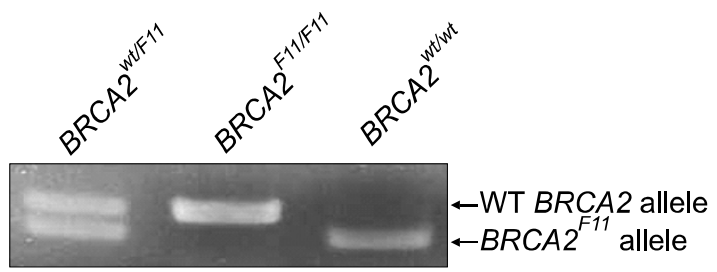

Figure 1. T-cell specific disruption of $B R C A 2$ using Cre-loxP recombination system. (A) Schematic representation of wild type and mutated $B R C A 2$ alleles. Two loxP sites as triangle flanks exon 11 of $B R C A 2$ $\left(B R C A 2^{F 11}\right)$. Recombination by Cre recombinase can induce excision of exon $11\left(B R C A 2^{\text {Del11 }}\right)$. Small arrows indicate PCR primer sites for mouse genotyping. (B) General genotyping pattern using PCR screening. Using primer $10 \mathrm{~F}$ and $10 \mathrm{R}$ in Figure $1 \mathrm{~A}$, wild-type allele is represented as 298 bp band, but band of $B R C A 2^{F 11}$ is shifted to $376 \mathrm{bp}$, since additional loxP sequences add up. ppressors is an important issue in cancer development, we observed the heterozygous mouse carefully. The result indicated that there was no sign of $\mathrm{LOH}$ in heterozygotes or did they have distinguishable phenotype compared to wild-type (data not shown). Heterozygous BRCA2 $2^{\text {wt/F11 }}$; Lck-Cre mice behaved normally just like the wild-type. Previous studies on several Brca2-mutant mice also indicate that the heterozygous do not have obvious phenotype compared to wild-type mice (Connor et al., 1997; Ludwig et al., 1997; Sharan et al., 1997; Suzuki et al., 1997; Friedman et al., 1998; Cheung et al., 2002).

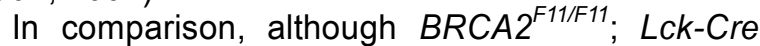
mice survived to birth like the wild-type or the heterozygote, the survival rate was reduced in 70-week observation after birth (Figure 2). Survival rate of $B R C A 2^{F 11 / F 11}$; Lck-Cre mice were compared with those of wild-type, BRCA2 $2^{\text {wt/F11 }}$; Lck-Cre, Lck-Cre, and $B R C A 2^{F 11 / F 11}$. Unless homozygous BRCA2 $2^{F 11 / F 11}$ mice are crossed with Lck-Cre mice, BRCA2 allele is not disrupted, therefore all the others could serve as controls. When the survival rate was monitored, we found that only 2 mice out of 40 control mice died when monitored for 70 weeks from birth. Death in control mice were observed at 32 weeks and 58 weeks, respectively, therefore we concluded that they lived up to their normal life span. In comparison, the death rate observed was $25 \%$ for 70 -week survival in BRCA2 $2^{\text {F11/F11 }}$; Lck-Cre mice. The time points of death were widespread from 16 weeks to 66 weeks after birth (Figure 2).

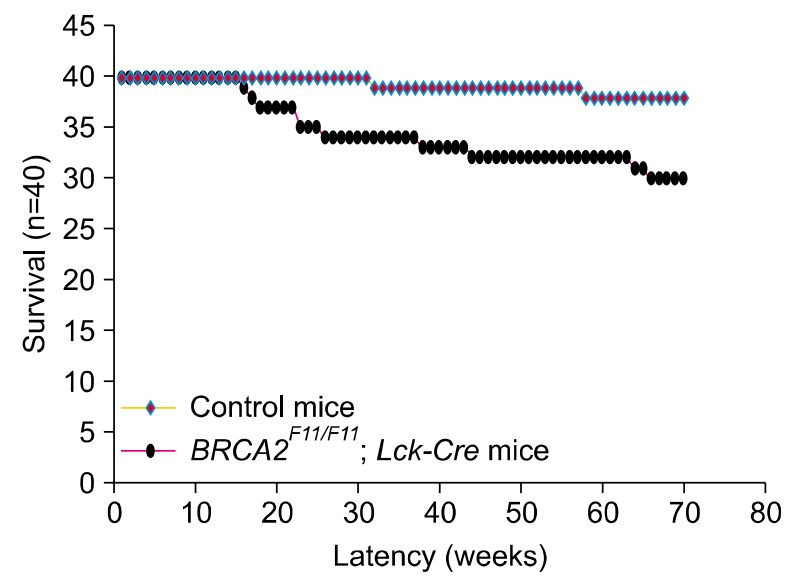

Figure 2. Survival rate of $B R C A 2 F^{11 / F 11}$; Lck-Cre mice and control mice. T-cell specific disruption of BRCA2 leads to low viability in comparison with that of control mice. While only 2 control mice were died naturaly (red square) during 70 weeks, 10 mice of BRCA2 ${ }^{\text {F11F F11; }}$; Lck-Cre mice died of thymic lymphoma (black circle). The timing of death is widespread (from 16 weeks to 66 weeks). Control mice include wild type mice, Lck-Cre mice and BRCA2 ${ }^{\text {wt/F11; }}$, Lck-Cre mice. Forty mice were analyzed each. 


\section{Development of thymic lymphomas in $\operatorname{Brca2}^{-11 / / 11}$ mice}

Next we analyzed for the cause of death in $B R C A 2^{F 11 / F 11}$; Lck-Cre mice. Because we engineered to disrupt Brca2 allele in the thymus in order to analyze cohort of thymic lymphomas, we first examined for any sign of abnormality in the thymus. Thymus from 26-week old wild-type mice was white, normal sized, and well attached to the heart (Figure $3 A)$. In contrast, thymus of dead BRCA2 $2^{\text {F11/F11; }}$ Lck-Cre mice of similar age were severely swollen and formed tough cellular adducts, which is the evident sign of lymphoma formation. This enlarged thymus was big enough to cover all around the heart (Figure 3B and C). Using primer 10F and 11R, we confirmed that Brca2 allele was effectively disrupted from the thymus of BRCA $2^{F 11 / F 11}$; Lck-Cre mice (Figure 3D). This result indicates that $B R C A 2^{F 11 / F 11}$; Lck-Cre mice died of thymic lymphoma. The causes for the death of two mice in control group were unrelated and did not show any sign of thymic lymphoma formation.

In order to analyze the $T$ cell populations in the thymic lymphomas, we cultured the suspension cells from the swollen thymus and analyzed for their CD4 and CD8 profiles (Figure 4). If they were thymic lymphomas, the CD4 and CD8 profiles will differ from that of normal thymus; it is likely that they form monoclonal populations. Thymus is the place for early T-cell development and maturation.
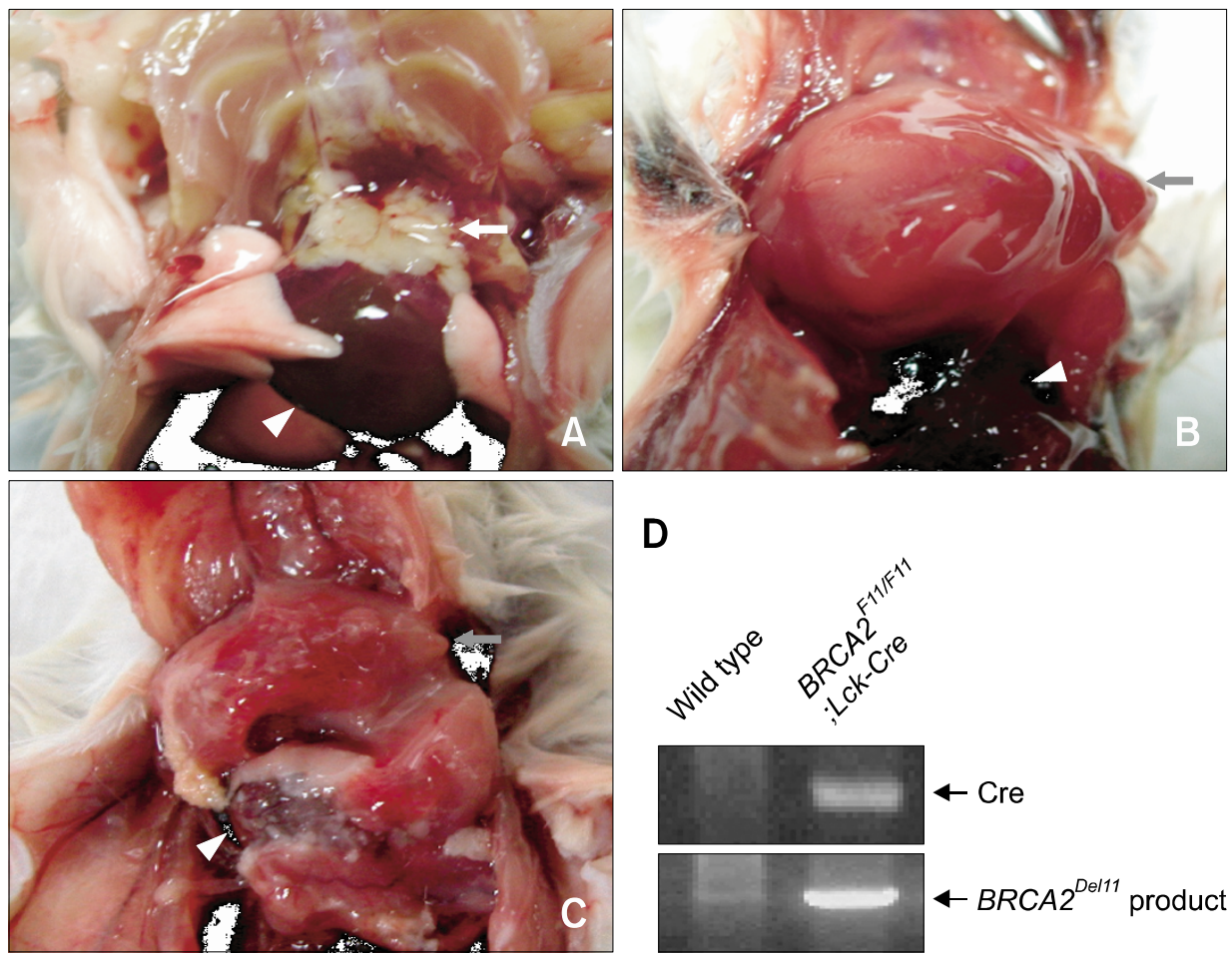

D

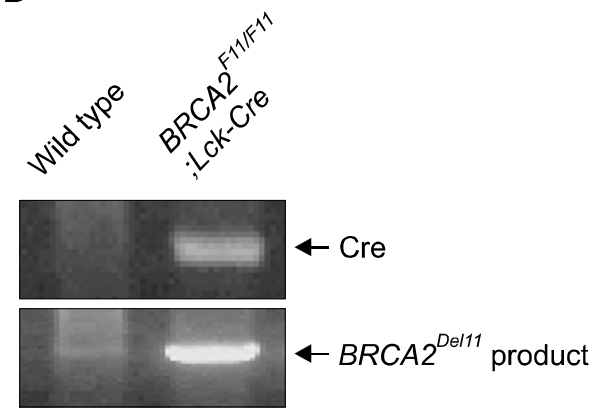

B

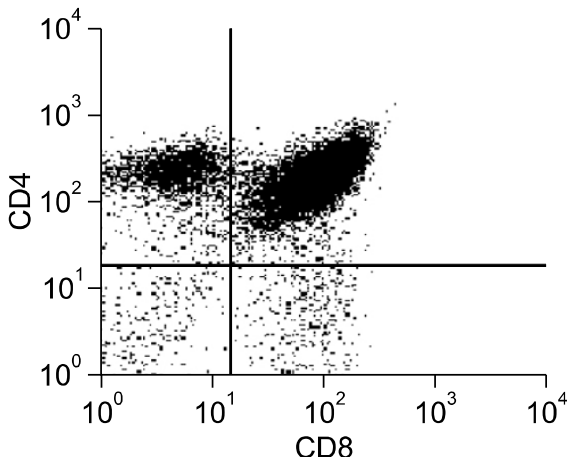

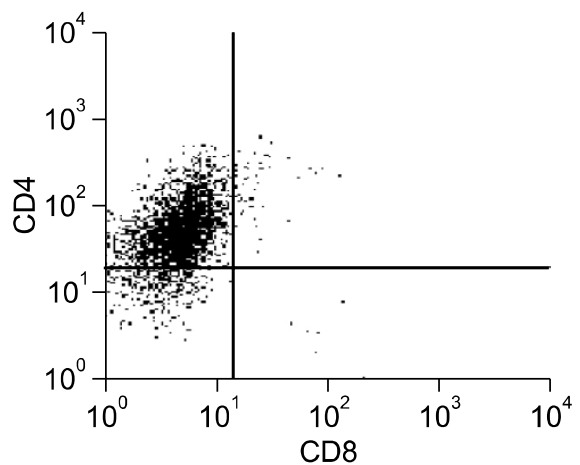

Figure 3. The thymuses of dead $B R C A 2^{F 11 / F 11 ;}$ Lck-cre mice were swollen and formed cellular adducts. All of 10 dead BRCA2 $2^{\text {F11/F11; }}$ Lck-cre mice developed thymic lymphomas. (A) Wild type thymuses are white and small. $(B, C)$ Thymuses of dead $B R C A 2^{F 11 / F 11}$; Lck-cre mice are swollen and large that they could cover all the way to surround the heart. These thymuses formed tough cellular adducts. (D) Using PCR screening with primer $10 \mathrm{~F}$ and $11 \mathrm{R}$ (Figure 1A), we confirmed that BRCA2 was disrupted along with the expression of Cre in thymic adducts of dead $B R C A 2^{F 11 / F 11 ; ~ L c k-c r e ~}$ mice. Triangle indicates heart, white arrow indicates normal thymus, and gray arrow indicates thymic adduct.

Figure 4. $C D 4$ and $C D 8$ property in thymus of dead BRCA2 F11/F11; Lckcre mouse. T cells in thymus from one of the dead BRCA2 $2^{F 11 / F 11 ; ~ L c k-c r e ~}$ mice were stained with CD4-FITC and CD8-PE for flow cytometric analysis. (A) Wild-type mice show normal profile of $T$ cell populations in the thymus; CD4 single positive, CD8 single positive, double positive and double negative cells. (B) $\mathrm{T}$ cells from dead $B R C A 2^{F 11 / F 11 ;}$; LckCre only contain $\mathrm{CD} 4$ single positive cells, suggesting that it is a monoclonal thymic lymphoma. 
Therefore, normal thymus contains all four types of $T$ cell repertoire; double positive, double negative, CD4 single popsitive or CD8 single positive cells. As anticipated, $T$ cells from wild-type mice showed all four populations (Figure 4A). In contrast, thymus from the dead BRCA2 $2^{\text {F11/F11 }}$; Lck-Cre mouse contained only CD4 single positive population, indicating that they were monoclonal tumor cells (Figure 4B). This result is in agreement with the previous report that thymoma from mice with truncated Brca2 allele contains monoclonal populations (Lee, 2003). Taken together, disruption of Brca2 allele in the thymus promotes $\mathrm{T}$ cell lymphoma.

\section{Discussion}

We have shown here that thymic $T$ cell-specific disruption of BRCA2 using Cre-loxP system leads to thymic lymphoma. Our results correlate with previous study in $\mathrm{Brca}^{\mathrm{Tr} / \mathrm{Tr}}$ mice where $\mathrm{Brca} 2$ allele is disrupted from the germ-line (Connor et al., 1997; Friedman et al., 1998; Patel et al., 1998; Lee et al., 1999). However, similar study by Cheung and colleagues has drawn a different conclusion (Cheung et al., 2002). The report suggested that T cell-specific disruption of $B R C A 2$ itself was not sufficient to induce $T$ cell lymphoma but exhibited aberrant chromosomes and increased apoptosis in response to DNA damage (Cheung et al., 2002). The difference may be due to different targeting site between two mice or different genetic background. The mice that were used in our experiments had targeted exon 11 of Brca2 (Jonkers et al., 2001), whereas the mice that were used by Cheng and colleagues had targeted exon 9 and 10 of Brca2 allele (Cheung et al., 2002). It should be emphasized that the very large exon 11 of Brca2 contains $6 \sim 8$ BRC repeats, which are implicated in Rad51 binding and modulating DNA repair. Therefore, we speculate that targeting exon 11 but not the other regions provided the basis of better survival and genetic instability. Also, we used FvB strain which has increased incidence for tumor formation (Woodworth et al., 2004), whereas Cheung and colleagues used 129/C57BL/6 or C57BL/6 (Cheung et al., 2002). Nevertheless, our results indicate that mutation in Brca2 in T cells predispose the cells to acquire higher rate mutations, leading to cancer. Therefore, these results indicate that the first hit in BRCA2 mutation is necessary for the initiation of genetic instability. Using this mouse model, we will be able to identify genes that cooperate with $B R C A 2$ on the road to cancer.

\section{Acknowledgment}

We thank Dr. Anton Berns (Netherland Cancer Institute, Netherlands) for kindly providing us with the Brca2 $2^{\mathrm{F} 11}$ and Lck-Cre mice. We are also grateful to H.W. Lee (Sungkyunkwan Univ., Kyunggido) and Dr. Y. Yun (Ewha Univ., Seoul) for providing services to maintain the mice. This work was supported from Biodiscovery Program (M10601000130-06N0100) from the Korean Ministy of Science and Technology and a grant endowed to $H$. Lee by Research Center for Functional Celulomics (R11-2005009-03004-0) of Seoul National University from KOSEF. This work was also supported by the second stage of the Brain Korea 21 Project in 2008. P-G Park is a recipient of Seoul Science Fellowship.

\section{References}

Cheung AM, Hande MP, Jalali F, Tsao MS, Skinnider B, Hirao A, McPherson JP, Karaskova J, Suzuki A, Wakeham A, You-Ten A, Elia A, Squire J, Bristow R, Hakem R, Mak TW. Loss of Brca2 and p53 synergistically promotes genomic instability and deregulation of T-cell apoptosis. Cancer Res 2002;62:6194-204

Connor F, Bertwistle D, Mee PJ, Ross GM, Swift S, Grigorieva E, Tybulewicz VL, Ashworth A. Tumorigenesis and a DNA repair defect in mice with a truncating Brca2 mutation. Nat Genet 1997;17:423-30

Daniels MJ, Wang Y, Lee M, Venkitaraman AR. Abnormal cytokinesis in cells deficient in the breast cancer susceptibility protein BRCA2. Science 2004;306:876-9

Davies AA, Masson JY, Mcllwraith MJ, Stasiak AZ, Stasiak A, Venkitaraman AR, West SC. Role of BRCA2 in control of the RAD51 recombination and DNA repair protein. Mol Cell 2001;7:273-82

Davies OR, Pellegrini L. Interaction with the BRCA2 C terminus protects RAD51-DNA filaments from disassembly by BRC repeats. Nat Struct Mol Biol 2007;14:475-83

Friedman LS, Thistlethwaite FC, Patel KJ, Yu VP, Lee H, Venkitaraman AR, Abel KJ, Carlton MB, Hunter SM, Colledge WH, Evans MJ, Ponder BA. Thymic lymphomas in mice with a truncating mutation in Brca2. Cancer Res 1998;58:1338-43

Gayther SA, Mangion J, Russell P, Seal S, Barfoot R, Ponder $B A$, Stratton MR, Easton D. Variation of risks of breast and ovarian cancer associated with different germline mutations of the BRCA2 gene. Nat Genet 1997;15:103-5

Huang Y, Myers MP, Xu RM. Crystal structure of the HP1EMSY complex reveals an unusual mode of HP1 binding. Structure 2006;14:703-12

Jonkers J, Meuwissen R, van der Gulden H, Peterse H, van der Valk M, Berns A. Synergistic tumor suppressor activity of BRCA2 and p53 in a conditional mouse model for breast cancer. Nat Genet 2001;29:418-25 
Lee $\mathrm{H}$, Trainer AH, Friedman LS, Thistlethwaite FC, Evans MJ, Ponder BA, Venkitaraman AR. Mitotic checkpoint inactivation fosters transformation in cells lacking the breast cancer susceptibility gene, Brca2. Mol Cell 1999;4:1-10

Lee $\mathrm{H}$. Impaired phosphorylation and mis-localization of Bub1 and BubR1 are responsible for the defective mitotic checkpoint function in Brca2-mutant thymic lymphomas. Exp Mol Med 2003;35:448-53

Ludwig T, Chapman DL, Papaioannou VE, Efstratiadis A. Targeted mutations of breast cancer susceptibility gene homologs in mice: lethal phenotypes of Brca1, Brca2, Brca1/ Brca2, Brca1/p53, and Brca2/p53 nullizygous embryos. Genes Dev 1997;11:1226-41

Patel KJ, Yu VP, Lee H, Corcoran A, Thistlethwaite FC, Evans MJ, Colledge WH, Friedman LS, Ponder BA, Venkitaraman AR. Involvement of Brca2 in DNA repair. Mol Cell 1998; 1:347-57

Pellegrini L, Yu DS, Lo T, Anand S, Lee M, Blundell TL, Venkitaraman AR. Insights into DNA recombination from the structure of a RAD51-BRCA2 complex. Nature 2002;420: 287-93

Sharan SK, Morimatsu M, Albrecht U, Lim DS, Regel E, Dinh C, Sands A, Eichele G, Hasty P, Bradley A. Embryonic lethality and radiation hypersensitivity mediated by Rad51 in mice lacking Brca2. Nature 1997;386: 804-10
Suzuki A, de la Pompa JL, Hakem R, Elia A, Yoshida R, Mo R, Nishina H, Chuang T, Wakeham A, Itie A, Koo W, Billia P, Ho A, Fukumoto M, Hui CC, Mak TW. Brca2 is required for embryonic cellular proliferation in the mouse. Genes Dev 1997;11:1242-52

Venkitaraman AR. A growing network of cancer-susceptibility genes. N Engl J Med 2003;348:1917-9

Venkitaraman AR. Tracing the network connecting BRCA and Fanconi anaemia proteins. Nat Rev Cancer 2004;4: 266-76

Woodworth CD, Michael E, Smith L, Vijayachandra K, Glick A, Hennings $H$, Yuspa SH. Strain-dependent differences in malignant conversion of mouse skin tumors is an inherent property of the epidermal keratinocyte. Carcinogenesis 2004;25:1771-8

Wooster R, Neuhausen SL, Mangion J, Quirk Y, Ford D, Collins N, Nguyen K, Seal S, Tran T, Averill D, et al. Localization of a breast cancer susceptibility gene, BRCA2, to chromosome 13q12-13. Science 1994; 265:2088-90

Yang H, Jeffrey PD, Miller J, Kinnucan E, Sun Y, Thoma NH, Zheng N, Chen PL, Lee WH, Pavletich NP. BRCA2 function in DNA binding and recombination from a BRCA2DSS1-ssDNA structure. Science 2002;297:1837-48 